\title{
Modern approach to facial skin defects reconstruction
}

\author{
Mateusz Kister', Katarzyna Borowska², Michał Gontarz', Bartłomiej Zoń3, \\ Grażyna Wyszyńska-Pawelec ${ }^{1}$, Jan Zapała', Barbara Jodłowska-Jędrych²
}

${ }^{1}$ Department of Cranio-Maxillofacial Surgery, Jagiellonian University Medical College, Rydygier Hospital, Cracow, Poland. ${ }^{2}$ Department of Histology and Embryology with Experimental Cytology Unit, Medical University of Lublin, Poland, ${ }^{3}$ Clinical Department of Plastic, Reconstructive Surgery and Burns Treatment, Military Medical Institute, Warsaw, Poland

Corresponding author: Mateusz Kister, mateuszkister@gmail.com

\begin{abstract}
Reconstruction of a facial defect is usually a challenging endeavor. The article aims to describe different types of flaps that might be used to restore such deformities- including their characteristics, indications and guidelines that should be followed in the reconstructive procedures.
\end{abstract}

Key words: Facial deformities; Flaps; Head and neck skin reconstruction

\section{INTRODUCTION}

Facial deformities are tremendously challenging conditions that in majority of cases require a reconstruction. They might be congenital or acquired and depending on their severity the following defects are distinguished: craniofacial, maxillofacial and dento-facial. In some instances, facial defects might be caused by the surgical intervention especially when the objective of a surgery is tumor resection.

Head and neck skin cancers constitute up to $12.2 \%$ of malignant tumors in Poland. Among all of them Basal Cell Carcinoma (BCC) is the most common. Due to its nature and prognosis, the most up to date protocol states that it should to be resected with at least $4 \mathrm{~mm}$ margin of healthy tissue [1]. The more malicious the tumor is, the wider margin of a healthy tissue needs to be resected. For example, for Squamous Cell Carcinoma (SCC) $1 \mathrm{~cm}$ of healthy tissue margin needs to be surgically removed and when it comes to malignant melanoma (MM) the value of $\mathrm{pT}$ indicating the size of the tumor is the determinant. Adequate margins of $4 \mathrm{~mm}$ for low-risk SCC and $6 \mathrm{~mm}$ for high-risk SCC have been demonstrated by direct tumor extension from the clinical margin, $\mathrm{BCC}$ 's more than $2 \mathrm{~cm}$ require margins as wide as $10 \mathrm{~mm}$, as do tumors with aggressive histological growth patterns, such as superficial BCC of the trunk and morpheaform lesions. A $2 \mathrm{~mm}$ margins yields a cure rate of $94 \%$ in small (less than $1 \mathrm{~cm}$ ) nodular BCC. Margins of 3-5 mm around a tumor and extending into subcutaneous fat are recommended for primary BCC less than $2 \mathrm{~cm}$ in diameter. Every type of tumor requires unique approach which is outlined by World Health Organization (WHO). Based on clinical experience those requirements are being constantly updated to get the best possible results.

The reconstruction of a facial defect is not an easy endeavor, as it involves a comprehensive understanding of human anatomy, facial defect itself- its location, prognosis; patient age and general condition. Moreover, the surgery itself requires a skillful operator capable of handling both hard and soft tissues to obtain good aesthetic and functional results [2].

The timing of the reconstruction depends, among other factors, upon the type of a facial defect. Many of the congenital facial deformities require reconstruction shortly after birth and might involve more surgical interventions in the future in order to accomplish the desire effect. The same goes for the surgical approach, as it can vary in invasiveness, aesthetic outcome, length of the procedure, safety of the patient and the long-term prognosis.

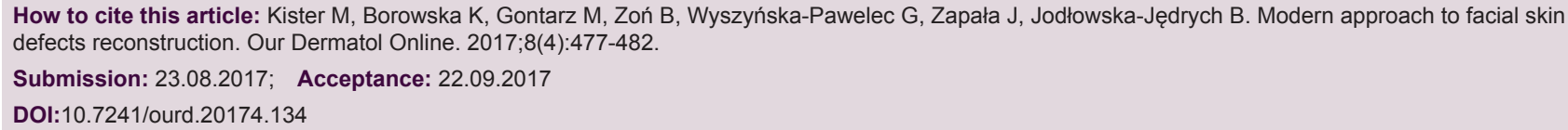


The correction of skin defects includes direct closure, local flaps, tissue grafts and free tissue transfers [3] (Fig. 1). Local skin flaps, when designed and performed in proper manner, allow prompt reconstruction with sufficient blood supply, fast healing time and acceptable visual outcome.

The term flap originates from the Dutch word 'flappe', meaning something suspended extensive and loose, attached only by one side [4]. The term 'flap' refers to a unit of soft tissue that is being transferred from one site to the other while keeping its own sufficient blood supply by the pedicle. When the unit of tissue is being transferred without its own blood supply then it is referred to as a tissue graft. Appropriate preparation of a skin graft takes more time than reconstruction involving skin flap, but the latter should potentially bring better aesthetic result [5].

The site from which the tissue is being harvested is often referred to as a donor site while the recipient site applies to the region to which the graft is transferred to. The history of flap surgery dates back to $600 \mathrm{BC}$ when nasal reconstruction by soft tissue flap harvested from patient's cheek has been mentioned for the first time [4].

Every surgeon aims for the best possible aesthetic outcome, thus the subunit principles should act as the foundation for a proper restoration. Attention should be paid to the unique colour, shade and texture of a skin, hair growth or subunit junctions as no single flap is ideal for each and every deformity. Every patient and each defect should be treated as an individual case for which a detailed analysis should be made and a long-term prognosis assessed. Skin flaps vary in shape and form and they range from a non-compound units

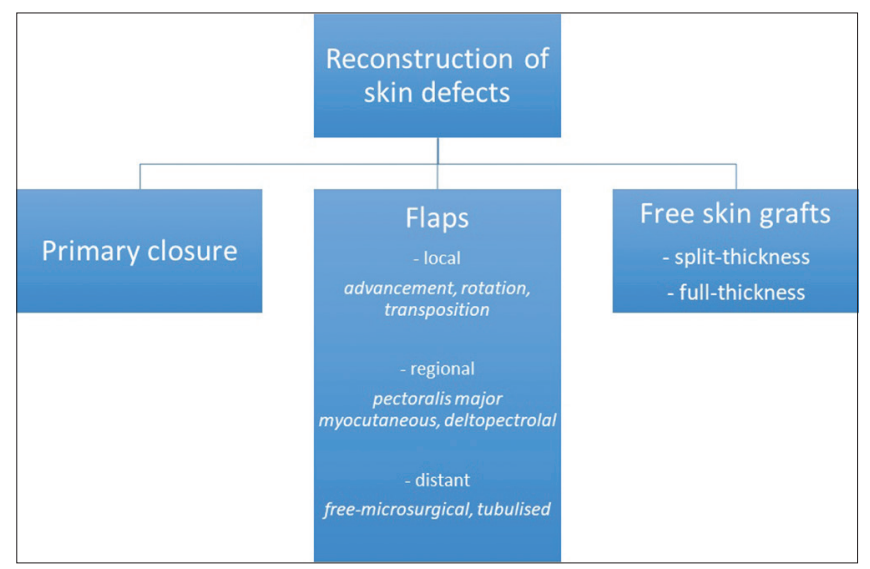

Figure 1: Types of skin reconstruction. of skin to being a composite of few different types of tissues including muscles, bone, fascia or fat.

It is not our objective to discuss in details different types of flaps as we aim to outline the general characteristics associated with different flaps based on our clinical experience and up to date literature.

\section{Guidelines for correct flap design}

Every reconstructive procedure requires detailed planning and comprehensive preparation as each case is unique. First thing that should be considered is a selection of the exact donor site that would provide sufficient tissue for the reconstruction [6]. Also, the way of closing the donor site should be considered at this point. The most common approaches include skin flap or skin graft transferred from distant or nearby location [7].

Other things, like requirements of shade, texture, size, presence of hair or its lack, size and thickness of skin should also be addressed in order to achieve the best possible functional and aesthetic outcome. Additionally, any possible risks for patient, probable side-effects and complications of surgery should be assessed. Next, preparation of the recipient site should follow.

Skin flaps are transferred from donor site to the recipient site sustaining their own blood supply. It is crucial that they retain their vascularity. That is why the most fundamental rule that needs to be obeyed when planning flaps in the head and neck region is the ratio between pedicle width and length of the flap which should be 1:2-1:6.

There are several ways by which a skin flap can be expanded. In some cases, an artificial expander can be placed underneath the designed flap. The other option is to line the flap with skin graft in a two-stage procedure, when second step will follow the first surgical intervention in approximately 14 to 21 days. The partial rising of a flap that is left in its original location will aid to its expansion and improvement of vascularity. This phenomenon is being explained by the occurrence of vasodilation that is said to last between 18 and 36 hours after the skin flap is raised. It should be noted, however, that vasodilation does not occur during the second surgery. Nonetheless, the technique of delaying the flap improves its blood supply and decreases the chances of ischemia and flap necrosis. That is why it is commonly used in more advanced and challenging reconstructions [8]. 


\section{Different types of reconstruction}

Simple excision that is followed by wound closure with released wound edges is considered to be one of the simplest procedures of removing skin tumors. This method usually brings satisfactory aesthetic outcomes [9,10] (Fig. 2). Even better results can be obtained when the course of the RSTL (relaxed skin tension lines) is obeyed. That type of lines was introduced by Borges and appears across whole body, mostly overlapping with natural folds with the exceptions of nose, lower lip and chin [11].

In most cases the simplest methods of reconstruction, including skin grafts, should be considered before more complex techniques are implemented. The most common donor sites for skin grafts harvested in the head and neck region are retro-auricular area and clavicular region, due to suitable shade and texture comparable to facial skin (Fig. 3).
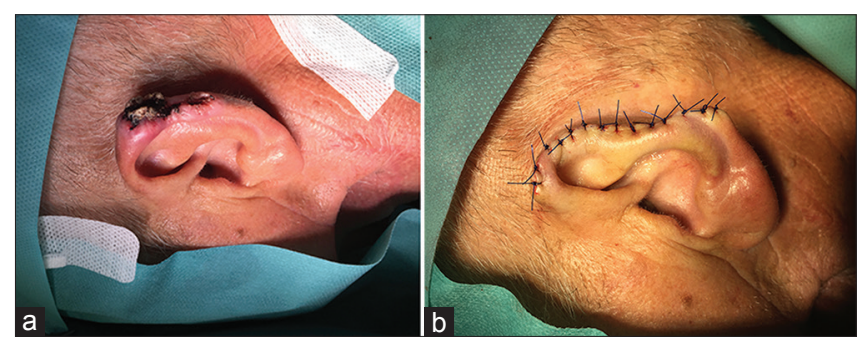

Figure 2: Simple excision technique presented on patient's left ear helix. Performed under local anesthesia.
Depending on the site to which the flaps are transferred, the regional and distant flaps are distinguished. The latter, which are used to cover nonadjacent deformities, might be raised as free microvascular flaps [10]. Free flaps are transferred from a donor site with own vascular pedicle and anastomosed to recipient vessels by microsurgical methods. The tubulised flap (Filatov flap), on the other hand, is moved from donor site to the other with its lateral borders sewn together which results in lower risk of infection and contraction of the flap. Its new blood supply will come directly from the distant pedicle of the flap. The regional flap is moved from donor to recipient site and pedicle is cut after two to three weeks $[9,10,12]$ (Fig. 4).

Local flaps are categorized according to the following criteria: composition, type of blood supply, method of movement. Composition refers to the tissues from which the flap is formed. As far as the blood supply is concerned, it can be random based on the rich subdermal vascular plexus or axial- getting its blood supply form a certain blood vessel. Yet, a lot of the head and neck flaps have random blood supply. The third feature - method of movement, is the most important. Based on the type of movement, advancement, interpolation, transposition and pivot/rotation various flaps are distinguished [9].

An advancement flap is relocated in only one directionin a straight path into the defect. It is commonly

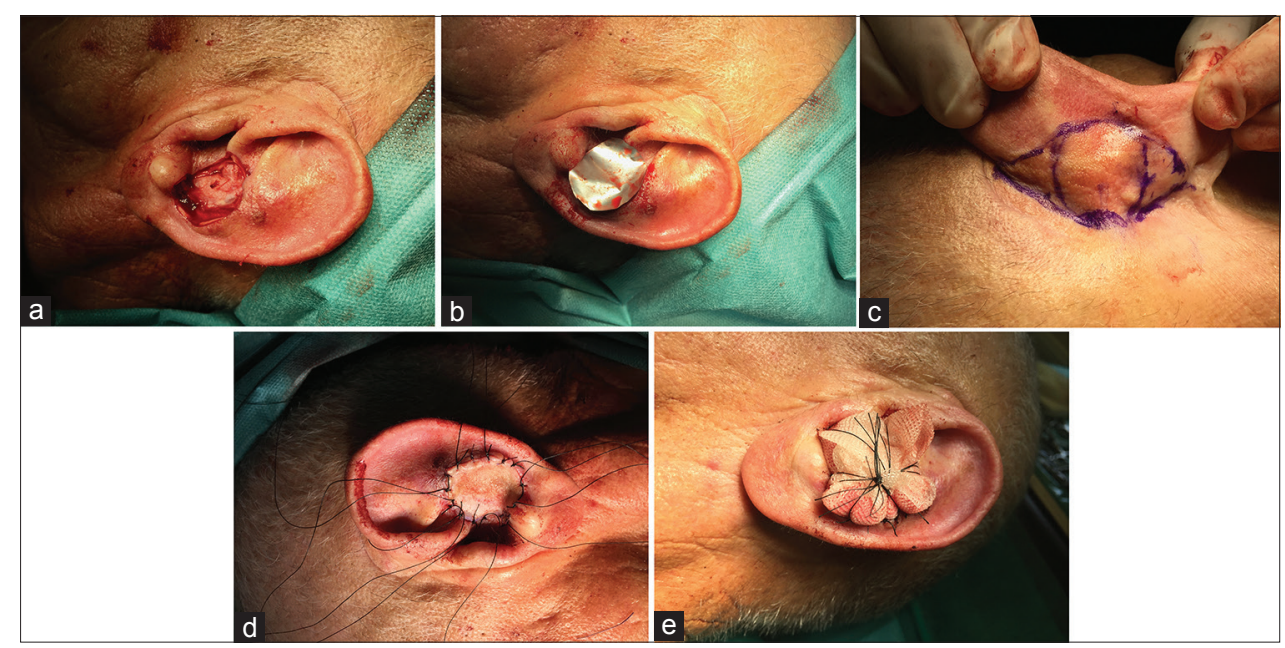

Figure 3: Full-thickness skin graft from retro-auricular site for defect reconstruction. Performed under local anesthesia. (a). The BCC excision with adequate margin. (b). The operative wound is measured using prepared matrix (usually sterile suture packing). (c). Using our matrix, the diameter of full-thickness skin graft is planned. Surgical blade is used to harvest a full-thickness skin graft. The wound outline is initially marked over the donor region. Usually its size is increased by $3-5 \%$ to compensate for primary contracture. Firstly, skin is incised and lifted with a skin hook. Any residual adipose tissue must be removed from the underneath of the graft since fat is poorly vascularized and can obstruct direct contact between the graft and the transplant area. Both full thickness and split grafts can be so called 'pie-crusted' that would let wound fluid to leak through the graft rather than cause its accumulation and thus preventing adherence. (d). The graft restoring wound is sutured using once short once long silk suture. (e). Sterile sponge covered in Xeroform net is tied using prepared sutures pressing the graft into the wound base. This would be removed after 5 days leaving all the sutures short for the next 5 up to 10 days. 


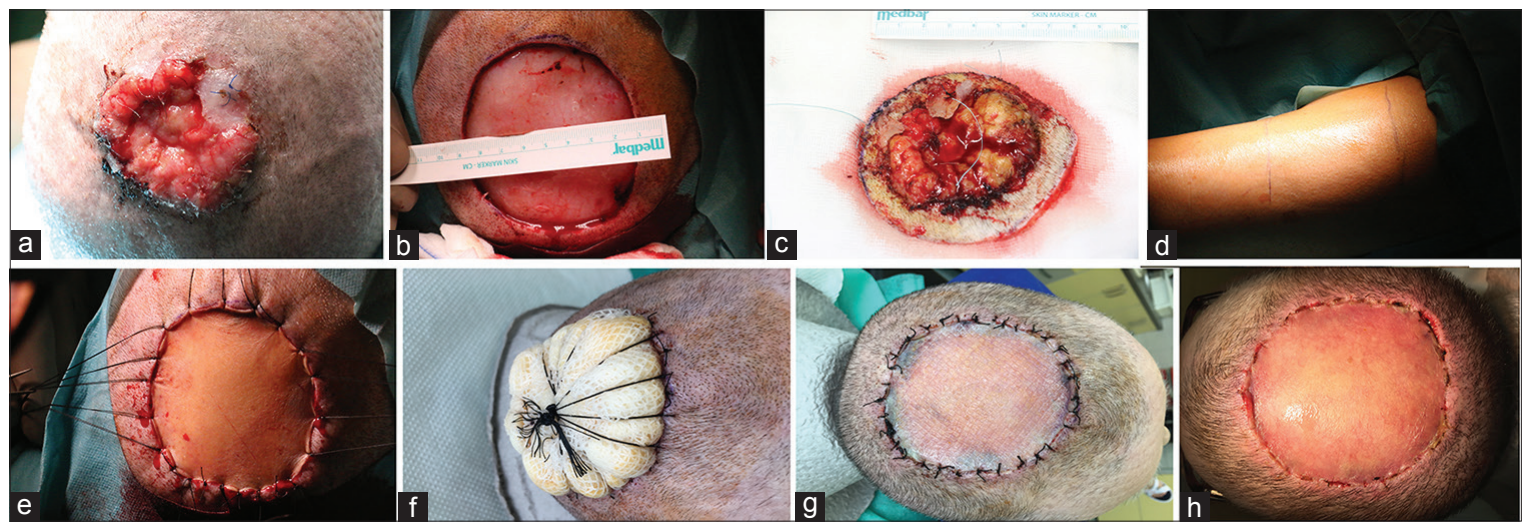

Figure 4: Split-thickness skin graft used for reconstruction of an extensive scalp defect. Performed under general anesthesia. (a). 90-year old patient with advance BCC of the scalp. (b). Post - excision of BCC. (c). Excision of basal cell carcinoma with the margin of macroscopically healthy tissue. (d). Left tight. The outline of the graft has been marked. Device called 'dermatome' is used for harvesting split-thickness skin graft where the depth and size of needed graft can be selected. Skin has been lubricated with paraffin prior to the usage of a device. (e). Placement of the skin graft. (f). "Tie-over" bolster dressings constructed from foam rubber Xeroform folded over moistened cotton balls or sponge is required for correct healing process. (g). Early removal of surgical dressing 5 days postop. (h). Removal of all remaining sttiches. Wound heals properly.

used in cheek, eyebrow, as well as forehead. It should be noted that Burrow's triangles excision at the base of the flap are crucial in order to prevent skin from 'dog-ear' formation [3]. A V-shaped flap is a unique type of an advancement flap used to restore a facial defect, especially on a cheek. After the donor site is closed, Y shaped suture line remains. The V-Y flap, on the other hand, is pushed rather than overextended into the defect and it causes a suture line of $\mathrm{Y}$ configuration [12].

Transposition flap is another type of flap. It should be longer than the defect, as transposition reduces its length. The flap moves laterally into the defect and the donor site might be closed either directly or indirectly. Examples of this kind of tissue restoration include Z-plasty, S-plasty, bilobed flap, the rhomboid flap, kite flap (Fig. 5).

The S- plasty in many instances can yield more aesthetically desired outcome for excision of a contour of a skin lesion without the necessity for a flap surgery. The procedure itself is quite simple: outline skin lesion to be removed by marking the $\mathrm{S}$-shape around the lesion, make the $\mathrm{S}$-shaped incision, secure hemostasis and place sutures. The advantages of this technique include, easier control of the wound tension, good aesthetic outcome and amplified length to width ration due to S-shaped incision [13].

The kite flap is a triangular flap attached to the subcutaneous tissue. It is one of the most efficient ways of reconstructing minor to medium sized cheek defects. This method is believed to have a practical and

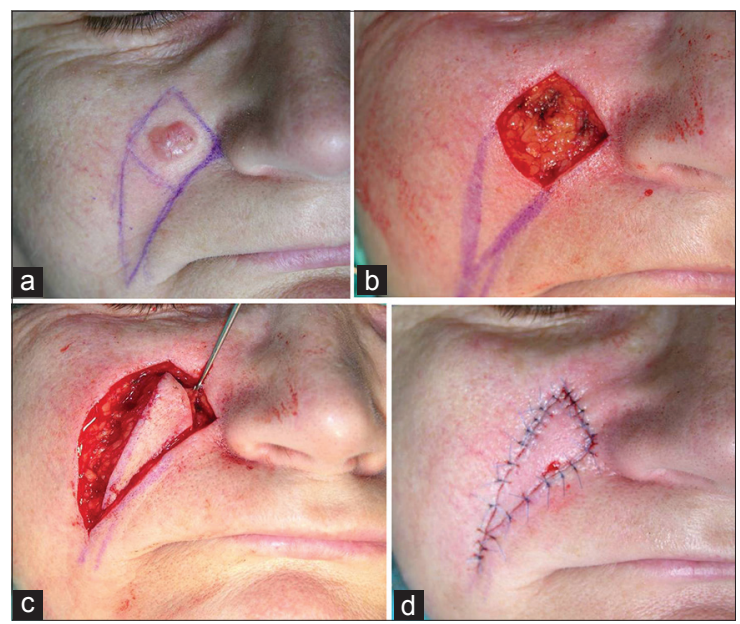

Figure 5: Island flap. Performed under local anesthesia. (Kite flap with a deep pedicle on the cheek used to reconstruct the operative defect with reshaping nasolabial fold. This kind of flaps supplies blood on vertical perforating vessels emanating from a deep source).

aesthetic advantage especially when compared to skin grafts or other local flaps in the cheek [14].

\section{Reconstruction of lip and nasal defects}

Lip and nasal defects are considered to be tremendously challenging to restore as they form the central part of the face.

In case of pathological lesions of the lower lip, W-Y excision often combined with vermilionectomy is used. The larger lesions, may require more invasive approach like Karapandzic, Bernard, Abbe, Estlander flaps. A recent 25-year retrospective study involving 2,152 patients with lip cancer revealed that 81 percent lesions occurred on the lower lip and higher prevalence was noted in males [12]. 
The purpose of the lip reconstruction is to restore the continuousness of the orbicularis oris muscle, mucous membrane and skin tissue. In order to achieve the best aesthetic outcome, the attention should be paid to the facial symmetry both at rest and during facial movements (Fig. 6).

Nasal deformities are among of the most perplexing facial defects to correct due to their complex structure, involvement of many components - mucosal lining, skin, bone, cartilage and vast variation of the skin tone and texture.

It is believed that the nasal tip and the lower third of a nose are the most challenging to restore. Probable restorative options include, skin graft, bi-lobe flap, single lobe flap, nasolabial flap, forehead flap or dorsal nasal flap [15]. The latter one, which consists of dorsal nasal skin from upper two thirds and glabella, is the most commonly used.

The dorsal nasal flap has many advantages: an excellent aesthetic result due to skin tone and texture match, acceptable scar and single stage method. Yet, the dorsal nasal flap cannot be used in defects of the nasal tip that are wider than $2 \mathrm{~cm}$ in diameter (Fig. 7).

The forehead flap and nasolabial fold flap are considered to be the gold standard for nasal reconstruction, as both have reliable pedicle and provide amount of tissue

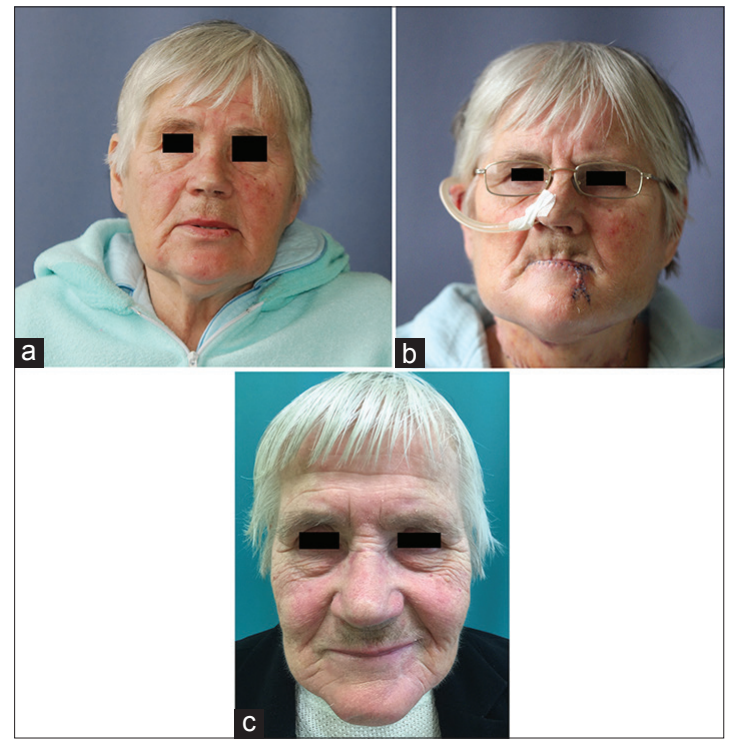

Figure 6: The W-Y plasty. Performed under general anesthesia. (a). One-stage SCC on lower lip excision combined with vermilionectomy procedure and selective bilateral neck dissection. (b). Patient is using nasogastric tube which provides quicker healing process. (c). Symmetry and maintained function 2 years after surgery sufficient for reconstruction of practically all nasal defects, including complex defects of nose and cheek (Fig. 8).

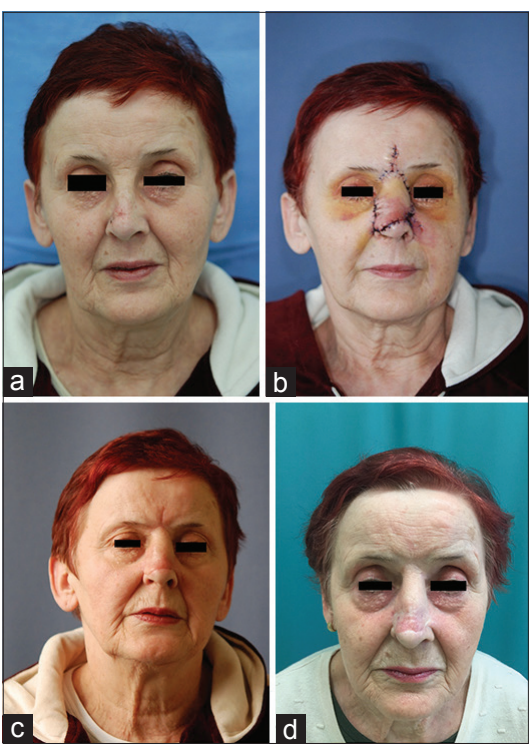

Figure 7: The Daniel Marchac's (lateral nasal) flap. Performed under general anesthesia. (a). 70-year old patient who underwent several procedures of simple non-radical excisions of BCCs from nasal skin in other medical units. (b). At our Department patient was qualified for the wide excision of postoperative scars and immediate flap reconstruction. In this type of reconstruction, the combination of V-Y and Z-plasty closure was used in glabellar region. (c). Short term result after sutures removal. (d). Result after 1 month.

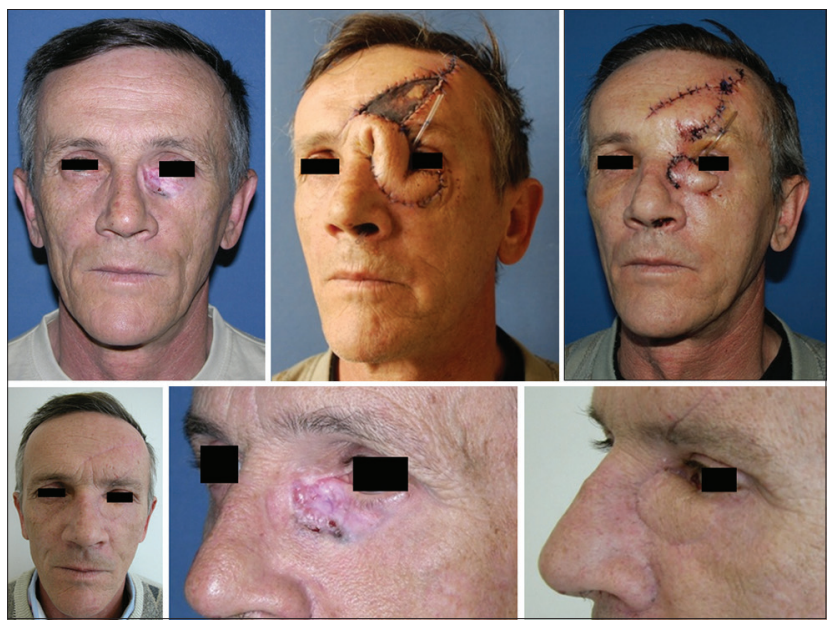

Figure 8: Supratrochlear artery (off-midline) forehead skin flap. Also, known as the Millard's flap, the surgeon who contributed significantly in its design. Performed under general anesthesia. (Two stage procedure containing surgical intervention followed by the carrier segment section after 14 days. In this case because of close infiltration to lower eye lid the lacrimal canaliculus was found and protected by plastic tube with open inside before making any cuts. The defect left by the carrier segment is closed in two layers and that left by the paddle is covered by split-thickness skin graft taken from thigh).

Photographies have been originally published in Zapała J, Wyszyńska-Pawelec G. "Wybrane Zagadnienia z Onkologii Głowy i Szyi“. 


\section{Examining the skin flap}

Once a flap has been successfully planned and implemented, the attention should be paid to monitoring its viability. This principle applies to every kind of facial flap. A prompt identification of ischemia and blood congestion, is crucial in averting subsequent flap necrosis and flap failure. Clinical observation is considered to be the best method to evaluate a skin flap. Based on the color of a flap the initial assessment might be made, as a very pale flap may indicate arterial deficiency, whereas a blue one - a failure of venous outflow. Additionally, an evaluation of a bleeding time after puncturing the flap with a small needle is still assumed to be one of the most trustworthy methods of clinical evaluation $[10,16,17]$.

Objective tests, like pH monitoring, Doppler ultrasound [17], surface temperature monitoring, thermography $[16,18]$ or transcutaneous oxygen tension (pO2) $[18,19,20]$ can be helpful in early detection of flap ischemia [17-19].

\section{CONCLUSION}

The aim of reconstruction of facial skin defects is to obtain the best possible aesthetic and functional result. Every case should be approached in an individual manner as no two patients, nor two facial defects are the same. Although we have described many various flaps, based on our clinical experience and it can be concluded that local flaps provide the most satisfactory results and should be considered as the first choice for the reconstruction of facial defects.

The success of the restorative surgery not only depends on the selected technique, or the type of flap used, but also on the skills of a surgeon performing the reconstructive procedure.

Through the last few decades rapid progress in microsurgical techniques is observed and free tissue transplants can now be used more frequently in reconstruction of skin defects. Although, free flaps enable to reconstruct large defects, their colour and texture differ from facial skin.

\section{REFERENCES}

1. Rao JK, Kaustubh Sharad Shende. Overview of local flaps of the face for reconstruction of cutaneous malignancies: single institutional experience of seventy cases. Department of Plastic Surgery, SMS Medical College. Jaipur, Rajasthan, India. 29-Dec2016.

2. Houseman N, Taylor G, Pan W. The angiosomes of the head and neck: anatomic study and clinical applications. Department of Plastic Surgery, Royal Melbourne Hospital. Melbourne, Australia. 2000.

3. Clark J, Wang T. Local Flaps in Scar Revision. Facial Plast Surg. 2001;17:295-308.

4. Chrysopoulo M. Tissue Flap Classification. Consulting Staff, Plastic, Reconstructive \& Microsurgical Associates of South Texas, USA. Sep 01, 2015.

5. Nakajima H, Fujino T, Adachi S. A new concept of vascular supply to the skin and classification of skin flaps according to their vascularization. Ann Plast Surg. 1986;16:1-19.

6. Walter Ernest O'Neil Yeo- One of the first people to undergo Plastic Surgery. The Yeo Society. 28 August 2008.

7. Lamberty H, Healy C. Flaps: Physiology, principles of design, and pitfalls. In: Cohen M, ed. Mastery of Plastic and Reconstructive Surgery. Vol 1. Boston: Little, Brown and Co. 1994:56-70.

8. Ghali S, Butler P, Tepper O, Gurtner G. Vascular delay revisited. Plast Reconstr Surg. 2007;119:1735-44.

9. Mathes S, Hansen S. Flap Classification and Applications. Plastic Surgery. 2nd ed. Philadelphia: Saunders; 2005. Vol. I: General Principles: 365-482/Chapter 16.

10. Fisher J, Gingrass M. Basic principles of skin flaps. Georgaide GS, Riefkohl R, Levin LS, eds. Textbook of Plastic, Maxillofacial, and Reconstructive Surgery. Baltimore: Williams and Wilkins; 1997. 19-28/ Chapter 4.

11. Borgese A. Relaxes skin tension lines (RSPL) versus other skin lines. Plast Reconstr Surg. 1984;73:144-50.

12. Cronin T. The V-Y rotational flap for nasal tip defects. Ann Plast Surg. 1983;11:282-8.

13. Sebastian S, Bang R, Padilla S. A simple approach to the s-Plasty in cutaneous surgery. Dermatol Surg. 2009;35:1277-9.

14. Ebrahimi A, Motamedi M, Koushki E, Nejadsarvari N. Applications of Kite Flap in Reconstruction of Cheek Defects after Tumor Excision. Maced J Med Sci. 2012;5:313-6.

15. Converse J. Introduction to plastic surgery. In: J.M. Converse (Ed.) Reconstructive plastic surgery. Vol. 1. $2^{\text {nd }}$ edition. WB Saunders, Philadelphia; 1977:3-68.

16. Levinsohn D, Gordon L, Sessler D. Comparison of four objective methods of monitoring digital venous congestion. J Hand Surg [Am]. 1991;16:1056-62.

17. Solomon G, Yaremchuk M, Manson P. Doppler ultrasound surface monitoring of both arterial and venous flow in clinical free tissue transfers. J Reconstr Microsurg. 1986;3:39-41.

18. Jones B, Mayou B. The Laser Doppler flowmeter for microvascular monitoring: a preliminary report. Br J Plast Surg. 1982;35: 147-9.

19. Sloan G, Sasaki G. Noninvasive monitoring of tissue viability. Clin Plast Surg. 1985;12:185-95.

20. Serafin D, Lesesne C, Mullen R, Georgiade N. Transcutaneous PO2 monitoring for assessing viability and predicting survival of skin flaps: experimental and clinical correlations. J Microsurg. 1981;2:165-78.

Copyright by Mateusz Kister, et al. This is an open-access article distributed under the terms of the Creative Commons Attribution License, which permits unrestricted use, distribution, and reproduction in any medium, provided the original author and source are credited.

Source of Support: Nil, Conflict of Interest: None declared. 